\title{
On rings with a unique proper essential right ideal
}

\author{
by \\ O. A. S. Karamzadeh, M. Motamedi and \\ S. M. Shahrtash (Ahvaz)
}

\begin{abstract}
Right ue-rings (rings with the property of the title, i.e., with the maximality of the right socle) are investigated. It is shown that a semiprime ring $R$ is a right ue-ring if and only if $R$ is a regular $\mathrm{V}$-ring with the socle being a maximal right ideal, and if and only if the intrinsic topology of $R$ is non-discrete Hausdorff and dense proper right ideals are semisimple. It is proved that if $R$ is a right self-injective right ue-ring (local right ue-ring), then $R$ is never semiprime and is Artin semisimple modulo its Jacobson radical ( $R$ has a unique non-zero left ideal). We observe that modules with Krull dimension over right ue-rings are both Artinian and Noetherian. Every local right ue-ring contains a duo subring which is again a local ue-ring. Some basic properties of right ue-rings and several important examples of these rings are given. Finally, it is observed that rings such as $C(X)$, semiprime right Goldie rings, and some other well known rings are never ue-rings.
\end{abstract}

1. Introduction. Throughout, as usual, all rings and subrings are associative with identity and all modules are assumed to be right unitary. The existence of maximal right ideals in rings and proper essential right ideals in non-semisimple rings is guaranteed by Zorn's lemma. Commutative rings with a unique maximal ideal (i.e., local rings) play an indispensable role in the study of commutative rings. But it seems (at least to us) that rings with a unique proper essential right ideal have never been systematically studied till now. In the literature, an essential right ideal is not assumed to be necessarily proper; see for example [1], [8]. Therefore rings with a unique essential right ideal are nothing but the Artin semisimple ones. We will observe shortly that rings with a unique proper essential right ideal, which we call right ue-rings, are interesting objects of study.

2000 Mathematics Subject Classification: Primary 16L30, 16L50; Secondary 16P20, $16 \mathrm{~N} 60$.

Key words and phrases: ue-ring, uem-ring, intrinsic topology, duo ring, isolated maximal right ideal.

The first author was supported in part by a grant from IPM. The authors would also like to thank the research council of Chamran University. 
Our main aim in this article is to study the structure of ue-rings and find some of their basic properties. We see trivially that right ue-rings are related to local rings, and also maximal right ideals which are direct summands (we call them isolated maximal right ideals; see [11], [12], [14], and [16]) appear naturally in the study of the structure of non-local right ue-rings. It is well known and very easy to prove that if every maximal right ideal of a ring $R$ is isolated, then $R$ is Artin semisimple. But if only one maximal right ideal of $R$ is non-isolated (we then call $R$ a right uem-ring), then the structure of $R$ is not known in general. Clearly, each right ue-ring is a right uem-ring. But the converse is not true in general. Indeed, if $X$ is an infinite completely regular topological space, then we prove that $C(X)$, the ring of real-valued continuous functions on $X$, is never a ue-ring. But $C(X)$ is a uem-ring if and only if $X$ is the one-point compactification of a discrete space. The study of these rings reveals that as a matter of fact, some important examples of rings known in the literature are ue-rings, and in fact the property of being a right ue-ring is responsible for some of their well known interesting properties. For example, Faith [8, p. 89] has constructed the first example of a regular (von Neumann) ring which is not a left V-ring (each simple left module is injective) but is a right $\mathrm{V}$-ring. We observe that this is a right ue-ring, and indeed any semiprime right ue-ring is a right V-ring. Also, we show that any local right ue-ring has a duo subring (every one-sided ideal is two-sided) which is again a right ue-ring.

An outline of this paper is as follows. After some preliminary results in Section 2, which are useful for the subsequent sections, the next section concentrates on right ue-rings, describing their properties and demonstrating their abundance. For example, in Theorem 16 we show that a ring $R$ is a right ue-ring if and only if it is either a local ring whose maximal right ideal has square zero, or a right uem-ring whose right socle is the non-isolated maximal right ideal. Semiprime right ue-rings are characterized in terms of their intrinsic topology and also via their cyclic modules; see Theorem 18. We observe that right ue-rings are Max-rings (i.e., every module over these rings has a maximal submodule). It is shown that $C(X)$, right self-injective semiprime rings and some other well known rings are never right ue-rings. Several examples of right ue-rings are given. Finally, in Section 4, we study duo ue-rings and show that they appear naturally in every local right ue-ring.

2. Preliminaries. Let $R$ be a ring and $M$ be a maximal right ideal of $R$. We call $M$ an isolated maximal right ideal provided that $M$ is a direct summand of $R$; see [12], [15], and [16]. We recall that a right ue-ring is a ring with a unique proper essential right ideal, and a right uem-ring is a ring with a unique essential maximal right ideal. Isolated maximal left ideals, left ue-rings and left uem-rings are defined similarly. We also define ue-modules 
in a natural way. Let $J, J_{0}$ denote respectively the Jacobson radical and the intersection of the isolated maximal right ideals in a ring $R . J_{0}$ is a two-sided ideal; see [11], [12]. If $R$ is a semiprime ring, then $J_{0}$ is also the intersection of the isolated maximal left ideals; see [12]. In [11] any ring $R$ is topologized (with the so-called intrinsic topology) by taking as a base of neighborhoods of zero those right ideals $A$ such that $R / A$ is a projective semisimple module. A right ideal $A$ of $R$ is open in this topology if and only if $A$ is the intersection of a finite number of isolated maximal right ideals; see [11, Proposition 1.3]. By a local ring we mean a ring $R$ with a unique maximal right ideal (equivalently, $R$ has a unique maximal left ideal). The reader is referred to [1], [8] for the basic results and undefined terms.

We begin with the following lemma from [17].

Lemma 1. $M$ is an isolated maximal ideal of a commutative ring $R$ if and only if $M$ is an isolated point in $\operatorname{Max}(R)$, the space of maximal ideals of $R$, with the Zariski topology.

Right semiartinian right V-rings, called right $S V$-rings, are extensively studied in [4]. We observe the following.

Proposition 2. The following are equivalent:

(1) $R$ is a right $S V$-ring.

(2) Every non-zero $R$-module contains a non-zero injective submodule.

(3) Every non-zero $R$-module contains a maximal submodule which is a direct summand (i.e., isolated).

Proof. (1) $\Leftrightarrow(2)$ is observed in [4].

$(1) \Rightarrow(3)$. Let $M \neq 0$ be an $R$-module. Since $\operatorname{Soc}(M) \neq 0, M$ contains a simple submodule, say $N$. As $R$ is a right V-ring, we infer that $N$ is injective, i.e., $M=N \oplus P$ for some submodule $P$. Clearly, $P$ is a maximal submodule.

$(3) \Rightarrow(1)$. Clearly, each non-zero $R$-module has non-zero socle, and hence is a Loewy module, which means that $R$ is semiartinian. To show that $R$ is a right V-ring, let $S$ be a simple $R$-module and $E(S)$ be its injective envelope. We claim that $E(S)=S$. Suppose not. Then by our hypothesis there exists a maximal submodule $P$ of $E(S)$ such that $E(S)=P \oplus Q$. Clearly, this is a contradiction, for $E(S)$ is indecomposable.

Lemma 3. In any ring $R$, we have $J \cap S=J_{0} \cap S$, where $S$ is the right socle of $R$.

Proof. Since every maximal right ideal is either isolated or essential, we infer that $J=J_{0} \cap A$, where $A$ is the intersection of all essential maximal right ideals. Thus $S \subseteq A$ and we are done.

For the proof of the following result see Propositions 1.1 and 1.3 in [11]. 
Proposition 4. Let $A$ be a right ideal in a ring $R$. Then the following are equivalent.

(1) $A$ is open in the intrinsic topology of $R$.

(2) $A+S=R$, where $S$ is the socle of $R$.

(3) $A$ is a finite intersection of isolated maximal right ideals.

Proposition 5. If $M$ is an isolated maximal right ideal of a ring $R$ and $x \notin M$, then $M^{\prime}=(M: x)=\{a \in R: x a \in M\}$ is also an isolated maximal right ideal.

Proof. We define $f: R / M^{\prime} \rightarrow R / M$ by $f\left(r+M^{\prime}\right)=x r+M$ for $r \in R$. Clearly, $f$ is an isomorphism. Thus $M^{\prime}$ is a maximal right ideal and since $R / M^{\prime}$ is projective, $M^{\prime}$ is isolated.

Corollary 6. $M$ is an isolated maximal right ideal of a ring $R$ if and only if $M / J_{0}$ is an isolated maximal right ideal of $R / J_{0}$.

Proof. Suppose $M / J_{0}$ is an isolated maximal right ideal in $R / J_{0}$. Then $M=e R+J_{0}$, where $e-e^{2} \in J_{0}$, i.e., $(1-e) M \subseteq J_{0}$. But clearly $1-e$ $\notin J_{0}$ implies that there exists an isolated maximal right ideal $M^{\prime}$ such that $1-e \notin M^{\prime}$. Thus $M=\left(M^{\prime}: 1-e\right)$ is an isolated maximal right ideal. The converse is evident.

We quote the following result from [13]; see also [12], [14].

Proposition 7. Let $R$ be a right self-injective ring. Then a maximal right ideal is projective if and only if it is isolated.

REMARK 8. If $R$ is a commutative self-injective ring or $R=C(X)$, then the previous result is still true if we replace "maximal right ideal" by "prime ideal"; see [13].

The following is a characterization of uem-rings.

Proposition 9. $R$ is a right uem-ring if and only if $R / \operatorname{Soc}_{r}(R)$ is a local ring.

Proof. If $R$ is local, then the statement is clear. Hence assume that $R$ is not local and $R$ is a right uem-ring. Let $M$ be the non-isolated maximal right ideal of $R$. Then clearly $S=\operatorname{Soc}_{r}(R) \subseteq M$ and $M$ is the only maximal right ideal containing $S$. Thus $R / S$ is a local ring. Conversely, if $R / S$ is local and $M / S$ is its unique maximal right ideal, then $M / S$ is non-isolated in $R / S$, i.e., $M$ is not isolated in $R$. Now we claim that each maximal right ideal different from $M$ is isolated. Let $P$ be such a maximal right ideal. Then $P$ is not essential, as otherwise $P \supseteq S$, which implies that $P=M$, a contradiction. Thus $P$ is isolated.

Let $I$ be an infinite set and $F$ be a field. Put $F_{i}=F$ for all $i \in I$ and $R=\prod_{i \in I} F_{i} / \sum_{i \in I} \oplus F_{i}$. Then Matlis has observed that no maximal 
ideal of $R$ is isolated; see [13]. We conclude this preliminary section with the following extension of Matlis's observation.

Proposition 10. Let $\left\{R_{i}\right\}_{i \in I}$ be an infinite family of non-zero rings. Then $R=\prod_{i \in I} R_{i} / \sum_{i \in I} \oplus R_{i}$ has no isolated maximal right (left) ideal.

Proof. We prove more, by showing that no non-zero principal right (resp. left) ideal is a minimal right (resp. left) ideal of $R$. To see this, let $a R$ be a non-zero right ideal of $R$. Then $a=\left\langle a_{i}\right\rangle+S$, where $S=\sum_{i \in I} \bigoplus R_{i}$, and $a_{i} \neq 0$ for $i \in T \subseteq I$ and $T$ is infinite. Now put $T=A \cup B, A \cap B=\emptyset$, where $A$ and $B$ are both infinite. Then if we put $x=\left\langle x_{i}\right\rangle+S$, where $x_{i}=a_{i}$ for all $i \in A$ and $x_{i}=0$ for all $i \in B$, then clearly $a R \supset x R$, and we are done.

3. Right ue-rings. Before giving some examples of right ue-rings, we prove the following sequence of results about ue-modules.

Proposition 11. Let $R$ be a ring. Then the following statements are equivalent.

(1) $R$ is a right ue-ring.

(2) The right socle of $R$ is a maximal right ideal of $R$ and $J^{2}=0$.

(3) Each right ideal of $R$ is either semisimple or a direct summand of $R$ and $R$ is not Artin semisimple.

Proof. (1) $\Rightarrow(2)$. Clearly the right socle of $R$, say $S$, is the unique essential right ideal of $R$. Let $M \supseteq S$ be a maximal right ideal. Then $M$ is essential, i.e., $M=S$. Now, $J \subseteq S$ and $S J=0$ implies that $J^{2}=0$.

$(2) \Rightarrow(3)$. If $A$ is a right ideal of $R$, then there exists a right ideal $B$ such that either $A \oplus B$ is proper essential or $A \oplus B=R$. Clearly, if $A \oplus B$ is proper essential, then $A \oplus B$ is the socle, i.e., in this case $A$ is semisimple. Finally, it is evident that $R$ is not Artin semisimple.

$(3) \Rightarrow(1)$. Let $E$ be any essential right ideal. Then by (3), $E$ must be semisimple, therefore $E$ must be the right socle.

COROLlary 12. Each semiprime right ue-ring is right hereditary.

Proof. Evident.

Remark 13. Proposition 11 is also true for ue-modules.

COROLlaRY 14. If $M$ is a ue-module over a ring $R$, then for any proper non-zero submodule $N$ of $M$, either $M / N$ is semisimple, or $M / N$ is a uemodule and $N$ is semisimple.

Proof. If $M / N$ is not semisimple, then $M / N$ has a proper essential submodule $P / N$. Clearly, $P$ is essential in $M$, i.e., $P=S=\operatorname{Soc}(M)$. This means that $M / N$ has a unique essential submodule, namely, $S / N$. Thus $M / N$ is a ue-module and $N \subseteq S$ is semisimple. 
Proposition 15. A non-semisimple R-module $M$ is a ue-module if and only if for each submodule $N$ of $M$, either $N$ is a direct summand of $M$ or $N$ is semisimple. Moreover, if $N$ is non-semisimple, then $N$ is a ue-module and $M / N$ is semisimple.

Proof. The first part is Remark 13. Now, let $M=N \oplus P$, where $N$ is not semisimple. Clearly, $N$ is a ue-module, for otherwise $M$ contains more than one essential submodule, which is not possible. Now it remains to show that $P$ is semisimple. To see this, assume by way of contradiction that $P \not \subset \operatorname{Soc}(M)=S$. Then $M=P+S$. Letting $S=P \cap S \oplus Q$, i.e., $M=P \oplus Q$, shows that $M / P \cong Q \cong N$ is semisimple, a contradiction, and therefore $P$ must be semisimple.

Next, we characterize right ue-rings.

TheOREM 16. A ring $R$ is a right ue-ring if and only if either $R$ is a local ring with unique maximal right ideal $M$ such that $M^{2}=0$, or $R$ is a right uem-ring and the non-isolated maximal right ideal is the socle of $R$.

Proof. Let $R$ be a right ue-ring and $S$ be its right socle. If $R$ is a local ring with the unique maximal right ideal $M$, we must have $S=M$. But $S J=0$ implies that $M^{2}=0$. If $R$ is not local, then each maximal right ideal except the right socle must be isolated. Conversely, if $R$ is a local ring with the unique maximal right ideal $M$ such that $M^{2}=0$, then $\operatorname{Ann}_{r}(x)=M$ for all $0 \neq x \in M$. Thus each $x R$ is a minimal right ideal, so $M$ is the socle of $R$ and we are done by Proposition 11. Finally, if $R$ is a right uem-ring whose right socle is a maximal right ideal, the proof is evident.

The next proof shows that each right ideal of a reduced right ue-ring is two-sided.

Corollary 17. Let $R$ be a reduced (i.e., with no non-zero nilpotent element) right ue-ring. Then each non-semisimple right ideal contains all minimal right ideals except finitely many.

Proof. Let $S=\sum_{i \in I} \bigoplus A_{i}$ be the right socle of $R$. Since $R$ is semiprime, each minimal right ideal $A_{i}$ is of the form $A_{i}=e_{i} R, i \in I$, where $e_{i}$ is a central idempotent. Now let $A$ be a right ideal of $R$ which is not semisimple. Then by Proposition $15, A$ is a direct summand of $R$ and $R / A$ is a semisimple $R$-module. Therefore $A$ is a finite intersection of maximal right ideals $M_{k}, k=1, \ldots, n$. But by Theorem 16, each $M_{k}$ is isolated, for $A$ is not semisimple. Now it is clear that $M_{k}=\left(1-e_{i_{k}}\right) R$ for each $k$ and some $i_{k} \in I$. Since $e_{i} e_{j}=0$ for all $i \neq j$, we immediately infer that $e_{j} \in M_{k}$ for $j \neq i_{k}$. As $A=\bigcap_{k=1}^{n} M_{k}$, we see that $e_{j} \in A$ for all $j \neq i_{1}, \ldots, i_{n}$. This means that $A_{j} \subseteq A$ for $j \neq i_{1}, \ldots, i_{n}$, and we are done. 
In [6] right V-rings $R$ with non-finitely generated right socle, say $S$, such that $R / S$ is a division ring are characterized. We note that these are nothing but semiprime right ue-rings which are nicely characterized as follows.

THEOREM 18. The following statements are equivalent for a semiprime ring $R$.

(1) $R$ is a right ue-ring.

(2) The intrinsic topology of $R$ is a non-discrete Hausdorff topology and a dense proper right ideal must be semisimple.

(3) $R$ is a regular, right $V$-ring and $R / \operatorname{Soc}(R)$ is a division ring with $\operatorname{Soc}(R) \neq 0$.

(4) Each cyclic R-module is either non-singular or injective, and does not have a finite Goldie dimension.

Proof. (1) $\Rightarrow(2)$. If $S$ is the right socle of $R$, then we know that $S J=0$. Hence $(S \cap J)^{2}=0$, i.e. $S \cap J=0$, because $R$ is semiprime. This implies that $J=0$. Now by Lemma 3 , we have $S \cap J_{0}=S \cap J=0$, i.e., $J_{0}=0$ and the intrinsic topology of $R$ is Hausdorff (note that $J_{0}=\overline{\{0\}}$, see also [11]). Now let $I$ be a dense proper right ideal. Then by Proposition 15, either $I$ or $R / I$ is semisimple. Assume that $I$ is not semisimple but $R / I$ is, and seek a contradiction. Since $R / I$ is cyclic, it is Artin semisimple, i.e., $I$ is a finite intersection of maximal right ideals. Each of them is isolated, for $I$ is not contained in the right socle. Thus by Proposition 4, $I$ is an open ideal, and hence closed, which is a contradiction.

$(2) \Rightarrow(3)$. We claim that $R$ is a right ue-ring. To see this, it suffices to show that each proper essential right ideal $E$ is dense. As the right socle, say $S$, is dense (see [11]), we infer immediately that $E$ is also dense. Now we recall that in semiprime rings the right socle is a regular ideal (see [4, Lemma $2.1]$ ), and since $R / S$ is a division ring (i.e., a regular ring), we immediately see that $R$ is a regular ring. Thus it remains to show that $R$ is a right $\mathrm{V}$-ring.

Let $M$ be a simple $R$-module. We must show that each homomorphism $f: I \rightarrow M$, where $I$ is a right ideal, can be extended to a homomorphism from $R$ to $M$. First, we observe that in any ring, $I$ can be taken to be essential, so in right ue-rings we can always take $I$ to be the right socle $S$. Now we consider two cases. First, suppose that $M=R / S$. Clearly, $S$ is generated by idempotents. If $e \in S$ is one, then consider $f: S \rightarrow R / S$, i.e., $f(e)=f(e) . e \in R / S . S=0$. Thus $f$ is the zero mapping and we are done. Secondly, let $M=R / I$, where $I$ is an isolated maximal right ideal. If $f: S \rightarrow M$ is a non-zero homomorphism then $\operatorname{Ker} f$ is a maximal submodule of $S$. But $S$ is semisimple, i.e., $S=\operatorname{Ker} f \oplus A$. This implies that $A$ is a minimal right ideal and therefore $A=e R$, where $e=e^{2}$. But $f=f_{\mid A}$ and trivially $f_{\mid A}$ can be extended to a homomorphism from $R$ to $M$, and the proof is complete. 
$(3) \Rightarrow(4)$. Clearly $R$ is a right ue-ring and since $R$ is a regular ring, it does not have a finite Goldie dimension, for otherwise it is Artin semisimple, which is absurd. Let $R / I$ be a cyclic $R$-module. By Proposition 15 , either $I \subseteq S=\operatorname{Soc}(R)$ or $R / I$ is Artin semisimple. If $I \not \subset S$, then $R / I$ is Artin semisimple, i.e., $I$ is a finite intersection of isolated maximal right ideals. Now by Proposition 5, we immediately infer that $R / I$ is non-singular.

Hence assume that $I \subseteq S$. Then $S=I \oplus A$ for some right ideal $A$. Put $\bar{R}=R / I$ and $\bar{A}=(A+I) / I=S / I$. Now let $\bar{B}=(B+I) / I$ be an $R$-submodule of $R / I$ maximal with respect to the property that $\bar{A} \cap \bar{B}=0$. Then either $\bar{B}=0$, which means that $\bar{A}$ is essential in $R / I$, or $\bar{A}+\bar{B}=\bar{R}$, for $\bar{A}$ is maximal in $\bar{R}$ (note that $\bar{R} / \bar{A} \cong R / S$ ).

Now if $\bar{B} \neq 0$ and $\bar{R}=\bar{A} \oplus \bar{B}$, then clearly $\bar{A}$ is cyclic, and since $\bar{A}=S / I$ is semisimple, it is a finite direct sum of simple modules. Thus $\bar{A}$ is injective, because $R$ is a V-ring. We also note that $\bar{B} \cong \bar{R} / \bar{A}$ is simple, i.e., $\bar{B}$ is injective and therefore $\bar{R}=R / I$ is injective.

Finally, let $\bar{B}=0$, i.e., $\bar{A}$ is essential in $\bar{R}$. Then clearly, $\bar{A}=\bar{S}=S / I$ is the only proper essential submodule of $\bar{R}$, i.e., $R / I \supseteq \bar{A} \cong A$. But clearly $A$ is non-singular, for $R$ is regular, and since $\bar{A}$ is essential in $R / I$, we infer that $R / I$ is also non-singular.

$(4) \Rightarrow(1)$ follows from the main result of [6] (namely, Theorem 6).

REMARK 19. In the proof of part $(2) \Rightarrow(3)$ of the previous theorem, in order to prove the injectivity of simple modules we considered two cases. Observe that the proof of the second case works equally well for the first case. But in the first case we have actually shown something more, that is, that there is only the zero homomorphism.

The following is interesting.

Corollary 20. Let $R$ be a right ue-ring. Then each element of $R$ is either a left or right zero divisor or a right unit.

Proof. If $R$ is a local ring, then the assertion is clear. Let $0 \neq a \in R$ be an element which is not a right unit and assume that $R$ is not local. Let $M$ be a maximal right ideal of $R$ containing $a$. If $M$ is isolated, then we are done. If not, $M$ must be the right socle, and if $J \neq 0$, then $M J=0$ finishes the proof. Thus we may assume that $J=0$. Then by the previous theorem $R$ is regular, and the proof is complete.

REMARK 21. If $R$ is a right ue-ring, then each $R$-module is a Loewy module with Loewy length less than or equal to 2 .

Now we are ready to present some examples of right ue-rings.

1. Let $R$ be a ring with a maximal right ideal $M$ such that $M$ is twosided and non-idempotent (for example, let $R$ be a duo ring with the latter property for $M$ ). Then by Theorem $16, R / M^{2}$ is a local right ue-ring. Thus 
for any commutative ring $R$ with infinitely many non-idempotent maximal ideals we have infinitely many non-isomorphic (as $R$-modules) local ue-rings.

2. A right chain ring is a right ue-ring if and only if it has a unique proper non-zero right ideal; see also our Proposition 33.

3. Let $D$ be a division ring and

$$
R=\left\{\left[\begin{array}{ll}
a & b \\
0 & c
\end{array}\right]: a, b, c \in D\right\}=\left[\begin{array}{cc}
D & D \\
0 & D
\end{array}\right] .
$$

Then the right socle of $R$ is $S_{1}=\left[\begin{array}{ll}O & D \\ O & D\end{array}\right]$ and the left socle is $S_{2}=\left[\begin{array}{ll}D & D \\ O & O\end{array}\right]$. These are the only maximal right ideals of $R$. Now $S_{1}$ as a left ideal is a direct summand, but as a right ideal it is not even principal, i.e., $S_{1}$ is an essential right ideal. Similarly, $S_{2}$ is isolated as a right ideal and essential as a left ideal. Thus $R$ is both a right and a left ue-ring.

4. If $Q$ is a right quotient of a right ue-ring $R$ with respect to a set of regular elements of $R$, then $Q$ is also a right ue-ring. Thus, a semiprime right Goldie ring is never a right ue-ring.

5. Let $M$ be a non-semisimple Artinian $R$-module with composition length two. Then clearly $M$ is indecomposable and it is easy to see that $T=\operatorname{End}\left(M_{R}\right)$ is a right ue-ring.

6. Let $D$ be a division ring and

$$
R=\left\{\left[\begin{array}{ccccc}
a & b & 0 & \ldots & 0 \\
0 & a & 0 & \ldots & 0 \\
\vdots & \vdots & \vdots & \ddots & 0 \\
0 & 0 & 0 & \ldots & c \\
0 & 0 & 0 & \ldots & a
\end{array}\right]: \begin{array}{c}
a_{i i}=a \in D, a_{12}=b \in D, a_{n-1, n}=c \in D, \\
\text { all other entries are zero }
\end{array}\right\}
$$

Then the set of all elements of $R$ with zero diagonals is the unique maximal right ideal $M$ of $R$ such that $M^{2}=0$. Clearly, $R / M \cong D$ and $R$ is an Artinian ue-ring which is also a duo ring.

7. Let $F$ be a division ring and $T=\left\{\left\langle a_{i}\right\rangle \in \prod_{i=1}^{\infty} F_{i}: a_{i}=a_{j}\right.$ for all $i, j$, and $F_{i}=F$ \}. Put $R=S+T$, where $S=\sum_{i=1}^{\infty} \bigoplus F_{i}$. Then clearly $R$ is a strongly regular ring which is a ue-ring (note that $S$ is the socle of $R$ and $R / S \cong T)$.

8. Let $F$ be a division ring and $A=\sum_{i \in I} \oplus F_{i}$, where $F_{i}=F$ for all $i \in I$ and $I$ is an infinite set. Put $R=A \times F$ with coordinatewise addition and multiplication given by $\left(a_{1}, b_{1}\right)\left(a_{2}, b_{2}\right)=\left(a_{1} a_{2}+a_{1} b_{2}+a_{2} b_{1}, b_{1} b_{2}\right)$. Then $R$ is a right ue-ring which is also a strongly regular, right V-ring; see Theorem 18.

9. In [22, Definition 6.1], a ring $R$ is called a special ring if $R$ is a nonNoetherian semiprime ring whose right ideals are two-sided and the socle of $R$ is a maximal ideal. $A$ ring $R$ is called a T-ring if every Loewy module is a direct sum of certain submodules; see [22, Remark 4.1]. Now it is interesting 
to note that from Proposition 6.1 of $[22]$ we immediately infer that given any duo ring $R$, either $R$ is a T-ring or there exists an ideal $I$ such that $R / I$ is a semiprime ue-ring (i.e., by our Theorem $18, R / I$ is also a regular V-ring). This shows that duo rings behave nicely when we study ue-rings.

10. There are division ring extensions $G \leq F$ and $H \leq F$ with $\operatorname{dim}\left({ }_{G} F\right)$ $=\operatorname{dim}\left(F_{H}\right)=3, \operatorname{dim}\left(F_{G}\right)=\operatorname{dim}\left({ }_{H} F\right)=2$, and ring isomorphisms $\alpha$ : $F \rightarrow G$ and $\beta: F \rightarrow H$ (see [24]). Let $R=F \times F$ as abelian group and define $(a, b)(c, d)=(a b, \beta(a) d+b c)$, moreover, let $T=F \times F$ and define $(a, b)(c, d)=(a b, \alpha(a) d+b c)$. Then both $R$ and $T$ are local Artinian right ue-rings. These examples were constructed in [24] for other purposes.

11. Let $V$ be an infinite-dimensional vector space over a field $F$, and set $Q=\operatorname{End}_{F}(V)$. We can identify $F$ with the subring of $Q$ of all scalar transformations. Put $R=S+F$, where $S$ is the ideal consisting of all transformations with finite-dimensional range. The subring $R$ of $Q$ was considered for the first time by Faith (see [8, p. 89]); it is a regular ring which is not a left V-ring. However, it is actually a right V-ring; see [8]. It is well known that $R$ is a unit-regular ring and whenever $\operatorname{dim}_{F} V=a$, where $a$ is an infinite cardinal, then the Goldie dimension of $R$ is greater than or equal to $a$. We also observe that $R$ is a right ue-ring and hence a right $\mathrm{V}$-ring (see our Theorem 18). Again by Theorem 18 and the fact that $R$ is not a left $\mathrm{V}$-ring, we infer that $R$ is not a left ue-ring. To show that $R$ is a right ue-ring, it suffices to prove that $S$ is the right socle of $R$. But it is well known that $S$ is the socle of $Q$. Now let $A$ be a minimal right ideal of $Q$, and let $0 \neq a \in A$. We must show that $A=a R$. Now $\operatorname{Ann}_{r}(S)=\operatorname{Ann}_{l}(S)=0$ in $Q$ implies that $a S \neq 0$, i.e., $a S=A=a R$. But $R / S \cong F$, and therefore $S$ is a maximal right ideal of $R$.

Let us return to further results on right ue-rings.

Proposition 22. No infinite product of rings can be a right ue-ring, and $R=\prod_{k=1}^{n} R_{k}$ is a right ue-ring if and only if for some $k, R_{k}$ is a right ue-ring and $R_{j}$ is Artin semisimple for $j \neq k$.

Proof. If $R=\prod_{i \in I} R_{i}$, where $I$ is an infinite set, then clearly $E=$ $\sum_{i \in I} \bigoplus R_{i}$ is a proper essential right (left) ideal of $R$ which is not a maximal right ideal, i.e., $R$ is not a right ue-ring.

Now suppose $R=\prod_{k=1}^{n} R_{k}$ is a right ue-ring. Then each proper essential right ideal $I$ of $R$ is of the form $I=\prod_{k=1}^{n} I_{k}$, where each $I_{k}$ is either proper essential in $R_{k}$ or $I_{k}=R_{k}$ and $I_{k} \neq R_{k}$ for some $k$. This implies that we cannot have two $R_{k}$ with proper essential right ideal. Therefore for some $k$, $R_{k}$ must be a right ue-ring and $R_{j}$ must be Artin semisimple for all $j \neq k$. The converse is evident.

A ring $R$ is called a Max-ring if each non-zero $R$-module has a maximal submodule (some authors call it a B-ring; see [8]). 
In [18], it is shown that every module with Krull dimension which is also a Loewy module is Artinian. It is well known that Artinian modules over Max-rings are Noetherian; see [18]. These facts and Remark 21, together with the next result, imply that modules with Krull dimension over a right ue-ring are both Artinian and Noetherian.

Proposition 23. Every right ue-ring is a Max-ring.

Proof. First, assume that $R$ is a local right ue-ring with maximal right ideal $M$. Let $A$ be any non-zero $R$-module. Since $M$ is nilpotent we infer that $A \neq A M$. Hence $A / A M$ is a vector space over the division $\operatorname{ring} R / M$. Thus $A / A M$ and therefore $A$ has a maximal submodule.

Now, let $R$ be a non-local right ue-ring with right socle $S$. If $A$ is any non-zero $R$-module, then we consider two cases. First, suppose $A=A S$. Then $A$ is semisimple (note that for each $a \in A$ and each minimal right ideal $I$, either $a I=0$ or $a I \cong I$ is a simple submodule of $A$ ) and therefore $A$ has a maximal submodule. Secondly, suppose $A \neq A S$. Then $A / A S$ is a vector space over the division ring $R / S$. Thus $A / A S$ and therefore $A$ has a maximal submodule.

The following evident result shows that some mild finiteness condition on a ue-module $M$ forces $M$ to be both Artinian and Noetherian.

REMARK 24. If $M$ is a ue-module with finite Goldie dimension, then $M$ has a composition series.

We need the following lemma.

LEMMA 25. If $R$ is a right ue-ring, then the right singular ideal $Z_{r}(R)=$ $\left\{a \in R: \operatorname{Ann}_{r}(a)\right.$ is an essential right ideal $\}$ is nilpotent.

Proof. Let $x \in Z_{r}(R)$. We show that $x^{2}=0$. For if $x^{2} \neq 0$, then clearly $\operatorname{Ann}_{r}(x)=\operatorname{Ann}_{r}\left(x^{2}\right)=S$, where $S$ is the right socle of $R$, implies that $x R \cap \operatorname{Ann}_{r}(x)=0$, which is impossible. Thus $Z_{r}(R) \subseteq J$ and by Proposition 11 , we are done.

The following is now immediate.

Proposition 26. If $R$ is a commutative ue-ring, then $Z_{r}(R)=J$.

Proof. By the previous lemma, $Z_{r}(R) \subseteq J$. Conversely, if $R$ is a local ring, then $J=M$ is the unique maximal ideal with $M^{2}=0$, i.e., $M \subseteq Z_{r}(R)$ and we are done. If $R$ is a non-local ue-ring, then $S J=0$, where $S$ is the socle of $R$. Hence, $\operatorname{Ann}(x)=S$ for all $x \in J$, i.e., $J \subseteq Z_{r}(R)$.

The following is also interesting.

Proposition 27. If $R$ is a right ue-ring and $M$ is a non-zero $R$-module, then $Z_{r}(M)=\operatorname{Soc}(M)$ if and only if no simple submodule of $M$ is projective. 
Proof. If $0 \neq x \in Z_{r}(M)$, then $\operatorname{Ann}_{r}(x)$ is a proper essential right ideal, i.e., $\operatorname{Ann}_{r}(x)=S=\operatorname{Soc}_{r}(R)$ for all $0 \neq x \in Z_{r}(M)$. This implies that $Z_{r}(M)$ is a vector space over the division ring $R / S$. Hence $Z_{r}(M)$ is semisimple over $R$, i.e., $Z_{r}(M) \subseteq \operatorname{Soc}(M)$. Now let $A \subseteq M$ be a simple $R$-submodule of $M$ which is not projective, i.e., $A \cong R / S$. This shows that $A$ is singular and therefore $A \subseteq Z_{r}(M)$. Thus $Z_{r}(M)=\operatorname{Soc}(M)$. The converse is evident.

Some rings are never right ue-rings. For example, $R[x]$ and $R[[x]]$ are never ue-rings, because the right socles of both rings are zero (note that if $I$ is a non-zero minimal right ideal of $R[x]$ and $f \in I$ is of least degree with $\operatorname{deg} f=n$, then $I=I x^{n+1}$, i.e., $\operatorname{deg} f \geq n+1$, which is absurd; the proof for $R[[x]]$ is similar). Also, if $\left\{R_{i}: i \in I\right\}$ is an infinite set of rings, then $\prod_{i \in I} R_{i} / \sum_{i \in I} \oplus R_{i}$ is never a right (or left) ue-ring.

In what follows we are going to show that some other well known rings, namely, $C(X)$ and right self-injective regular rings, are never right ue-rings.

Proposition 28. If $R$ is a right self-injective right ue-ring, then $R / J$ is Artin semisimple.

Proof. If $R$ is local, then the assertion is clear. Now suppose $R$ is nonlocal. Then in view of Corollary $14, R / J$ is either semisimple or a right ue-module over $R$. But $R / J$ is regular by Theorem 18 (note that it is also well known that $R / J$ is regular and self-injective even without assuming $R$ is a ue-ring). Now suppose $R / J$ is a ue-module and seek a contradiction. Let $M / J$ be the unique proper essential right ideal of $R / J$ which is also a maximal right ideal and the right socle of $R / J$. Now clearly $M / J$ is the direct sum of minimal right ideals of $R / J$ which are generated by idempotents. Hence $M / J$ is projective and then Proposition 7 shows that $M / J$ is isolated, which is the desired contradiction.

The next result is now immediate.

COROLlary 29. A right self-injective regular ring is never a right uering.

The above corollary and Theorem 18 immediately yield the following.

Corollary 30. A right self-injective right ue-ring is never semiprime.

The reader is referred to [10] for some information on $C(X)$ for the next two results.

Proposition 31. An infinite completely regular topological space $X$ is the one-point compactification of a discrete space if and only if $C(X)$ is a uem-ring.

Proof. We recall that in any space $X, M_{x}=\{f \in C(X): f(x)=0\}$ is a maximal ideal, and $x$ is an isolated point of $X$ if and only if $M_{x}$ is an 
isolated maximal ideal (see [17]). Now if $X$ is the one-point compactification of a discrete space $D$, then each maximal ideal of $C(X)$ is fixed (i.e., of the form $M_{x}$; see [10]). Thus each maximal ideal of $C(X)$ is either of the form $M_{a}, a \in D$, which is isolated by what we have already shown at the beginning of the proof, or of the form $M_{x}$, where $x$ is the unique point which is not isolated, i.e., $M_{x}$ is not isolated.

Conversely, if $C(X)$ is a uem-ring, then each maximal ideal is isolated except one, say $M$. This shows that in $X$ we cannot have two non-isolated points, say $x_{1}, x_{2}$, for in that case we would have two non-isolated maximal ideals, $M_{x_{1}}, M_{x_{2}}$. Therefore there is only one non-isolated point $x$, i.e., $M=M_{x}$. But it is clear that each isolated maximal ideal is fixed (see [17]), i.e., all maximal ideals are fixed, which implies that $X$ is compact and therefore the one-point compactification of a discrete space.

Corollary 32. $C(X)$ is never a ue-ring.

Proof. If $X$ is finite, then $C(X)$ is a finite product of fields, and hence not a ue-ring. Now suppose $X$ is infinite and $C(X)$ is a ue-ring. Then by the previous proposition, $X$ is the one-point compactification of a discrete space, and in view of Theorem $18, C(X)$ is regular, i.e., $X$ is a $\mathrm{P}$-space (see $[10,4 . \mathrm{J}]$. But every compact $P$-space is finite (see $[10,4 . \mathrm{K}]$ ), which is a contradiction.

Before concluding this section, we note that Corollary 30 naturally raises the question if a right ue-ring can be right self-injective. The answer is trivially affirmative, for if $p \in \mathbb{Z}$ is a prime number, then $\mathbb{Z} /\left(p^{2}\right)$ is a selfinjective local ring with a unique non-zero ideal, namely $(p) /\left(p^{2}\right)$, i.e., a ue-ring (see Example 1). Motivated by this example we conclude this section with the following interesting observation.

Proposition 33. Let $R$ be a right self-injective local right ue-ring. Then $R$ has a unique non-zero left ideal.

Proof. Let $M$ be the unique maximal right ideal of $R$. It suffices to show that $M$ is the smallest non-zero left ideal of $R$. To this end, we prove that given any $0 \neq a \in M$, we have $M=R a$. Now $M^{2}=0$ implies that $\operatorname{Ann}_{r}(x)=\operatorname{Ann}_{l}(x)=M$ for all $0 \neq x \in M$. Thus let $0 \neq b \in M$ be an arbitrary element of $M$ and consider the homomorphism $f: a R \rightarrow b R$ defined by $f(a r)=b r$ for all $r \in R$ (note that $\operatorname{Ann}_{r}(a)=\operatorname{Ann}_{r}(b)$ ). The right self-injectivity of $R$ implies that $f$ can be extended to $R$, i.e., $f$ is multiplication by some element $0 \neq t \in R$. Hence $b=f(a)=t a \in R a$, i.e., $M=R a$.

4. Duo ue-rings. Duo rings, although non-commutative, have many properties of commutative rings. Every idempotent in these rings is central and prime ideals are completely prime and commute with each other 
(see [15]). These rings can be localized at their prime ideals. Unfortunately, this localization may not be a duo ring. Nevertheless, for a large class of duo rings, including Noetherian duo rings, and duo rings whose ideals commute, this localization is again a duo ring (see [5], [15]). As we have already observed in Example 9, duo rings are appropriate objects to consider when we are dealing with ue-rings. In this section we show that any local right ue-ring $R$ contains a duo subring $R_{d}$ which is a local ue-ring, and if $R_{d}$ is Artinian, then so is $R$. We also characterize Artinian duo rings which are ue-rings.

The following is interesting.

Proposition 34. Let $R$ be a duo ue-ring. Then the socle of $R$ is projective if and only if $R$ is regular (i.e., strongly regular: $\forall a \in R \exists b \in R$ with $\left.a=a^{2} b\right)$.

Proof. Suppose $S=\operatorname{Soc}(R)$ is projective. Note that $S J=J S=0$. We claim that $J=0$, which completes the proof in view of Theorem 18. So suppose $x \in J$ is non-zero and seek a contradiction. Clearly, $\operatorname{Ann}_{r}(x)=S$, i.e., $x R \cong R / S$. But $x R \subseteq S$, so $x R$ is a direct summand of $S$. This implies that $x R$ is projective, and hence the exact sequence $0 \rightarrow S \rightarrow R \rightarrow R / S \rightarrow 0$ shows that $S$ is a direct summand, which is a contradiction. The converse is evident.

Next, we show that duo ue-rings are zero-dimensional.

Proposition 35. If $R$ is a duo ue-ring, then each prime ideal in $R$ is maximal.

Proof. If $R$ is a local ring, then there is only one prime ideal and we are done. Hence assume that $R$ is non-local. If $J=0$, then by Theorem $18, R$ is regular and therefore each prime ideal is maximal. So assume $J \neq 0$. Then $S J=0$, where $S$ is the socle of $R$. Now for any prime ideal $P$ of $R$ we have either $S \subseteq P$ or $J \subseteq P$. If $S \subseteq P$, then $S=P$, i.e., $P$ is maximal. If $J \subset P$, then by Corollary 14, either $R / J$ is an Artin semisimple ring or a ue-ring. If $R / J$ is Artin semisimple, then $P / J$ and therefore $P$ is maximal, and if $R / J$ is a ue-ring, then by Theorem $18, R / J$ is regular, so again $P / J$ and therefore $P$ is maximal, and the proof is complete.

The next result shows that duo ue-rings appear naturally inside local right ue-rings.

TheOREm 36. Let $R$ be a local right ue-ring with $\operatorname{Soc}_{r}(R)=S$. Then $R$ contains a subring $R_{d}$ with socle $S$ such that $R_{d}$ is a duo ue-ring. Moreover, $R$ is right Artinian whenever $R_{d}$ is.

Proof. For each $t \in S$, we put $R_{t}=\{r \in R: r t=t r\}$. Clearly, $R_{t}$ is a subring of $R$ containing $S$. Now put $R_{d}=\bigcap_{t \in S} R_{t}$. We claim that $R_{d}$ has the required properties. 
First, we show that $R_{d}$ is a right ue-ring. It suffices to show that $S$ is the unique maximal right ideal in $R_{d}$. Therefore we must prove that each non-unit of $R_{d}$ belongs to $S$. To this end, we show that each non-unit $a \in R_{d}$ is in fact a non-unit of $R$, and this completes the proof. Suppose that $a \in R$ is a unit and seek a contradiction. For each $0 \neq t \in S$, we have $a t=t a$, i.e., $a^{-1} t=t a^{-1}$, which implies that $a^{-1} \in R_{t}$ for all $t \in S$, i.e., $a^{-1} \in R_{d}$, which is the desired contradiction.

To show that $R_{d}$ is a duo ring, we note that $a R_{d}=R_{d} a=R$ if $a \in R_{d}$ is a unit, and if $a \in S$, then $a R_{d}=R_{d} a$ by the definition of $R_{d}$. Hence $R_{d}$ is a duo ring.

For the proof of the last part, we note that in order that a local right ue-ring be right Artinian it suffices that its unique maximal right ideal be finitely generated. But the maximal right ideal $S$ is inside both rings $R$ and $R_{d}$, so $S$ being f.g. as an $R_{d}$-module implies that it is also f.g. as an $R$-module.

Remark 37. By the above proof, each $R_{t}$ is a local right ue-ring. Therefore if $R$ is not a duo ring, then for some $t \in S, R_{t}$ is a proper subring. Moreover, $R$ is right Artinian if some $R_{t}$ is.

REMARK 38. If the characteristic of a local right ue-ring $R$ is a prime number $p$, then $S+Z_{p}$ is a commutative subring of $R$ which is a local ue-ring, where $S$ is the socle of $R$. Moreover, $R$ has a maximal commutative ue-subring with socle $S$.

REMARK 39. Let $R$ be a local right ue-ring with $\operatorname{Soc}_{r}(R)=S$. For each $t \in R$, put $R_{t}=\{r \in R: r t \in t R$ and $t r \in R t\}$ and $R_{0}=\bigcap_{t \in S} R_{t}$. Then by applying the above proof we can show that $R_{0}$ and each $R_{t}$ are local right ue-rings and clearly $R_{0}$ contains $R_{d}$. Moreover, $R_{d}$ is contained in a maximal duo ue-subring of $R$ with socle $S$.

We conclude this section with the following observation, which characterizes Artinian duo ue-rings.

Proposition 40. $R$ is an Artinian duo ue-ring if and only if $R$ is the product of a local Artinian duo ue-ring and a finite product of division rings.

Proof. Let $R$ be an Artinian duo ue-ring. Then using the usual proof of the well known result on the decomposition of Artinian commutative rings (without any change), we show that $R$ is a finite product of local Artinian rings. Now by Proposition 22, we are done. The converse is evident.

Acknowledgements. The authors would like to thank the referee for a careful reading of this article. 


\section{References}

[1] F. W. Anderson and K. R. Fuller, Rings and Categories of Modules, Springer, 1992.

[2] E. Armendariz, Rings with dcc on essential left ideals, Comm. Algebra 8 (1980), 299-308.

[3] G. Baccella, A study of the socle of a ring, ibid., 889-909.

[4] -, Semiartinian V-rings and semiartinian von-Neumann regular rings, J. Algebra 173 (1995), 587-612.

[5] H. H. Brungs, Three questions on duo rings, Pacific J. Math. 58 (1975), 345-349.

[6] Dinh Van Huynh, J. K. Jain and S. R. López-Permouth, On a class of nonNoetherian V-rings, Comm. Algebra 24 (1996), 2839-2850.

[7] A. A. Estaji and O. A. S. Karamzadeh, On $C(X)$ modulo its socle, Comm. Algebra 31 (2003), 1561-1571.

[8] C. Faith, Algebra II, Ring Theory, Springer, 1976.

[9] L. Fuchs, Torsion preradicals and ascending Loewy series of modules, J. Reine Angew. Math. 239/240 (1969), 169-179.

[10] L. Gilman and M. Jerison, Rings of Continuous Functions, Van Nostrand, 1960.

[11] O. Goldman, A Wedderburn-Artin-Jacobson structure theorem, J. Algebra 34 (1975), 37-64.

[12] O. A. S. Karamzadeh, On maximal right ideals which are direct summands, Bull. Iran. Math. Soc. 18 (1983), 40-46.

[13] -, On a question of Matlis, Comm. Algebra 25 (1997), 2717-2726.

[14] - Projective maximal right ideals of self-injective rings, Proc. Amer. Math. Soc. 48 (1975), 286-288.

[15] —, On duo rings, Bull. Iran. Math. Soc. 9 (1982), 143-148.

[16] O. A. S. Karamzadeh and M. Motamedi, On the intersection of maximal right ideals which are direct summands, ibid. 18 (1983), 47-54.

[17] O. A. S. Karamzadeh and M. Rostami, On the intrinsic topology and some related ideals of $C(X)$, Proc. Amer. Math. Soc. 93 (1985), 179-184.

[18] O. A. S. Karamzadeh and A. R. Sajedinejad, On the Loewy length and Noetherian dimension of Artinian modules, Comm. Algebra 30 (2002), 1077-1084.

[19] N. K. Kim and Y. Lee, Armendariz rings and reduced rings, J. Algebra 223 (2000), $477-488$.

[20] J. N. Manocha, On rings with essential socle, Comm. Algebra 4 (1976), 1077-1086.

[21] W. K. Nichelson and J. F Watters, Rings with projective socle, Proc. Amer. Math. Soc. 102 (1988), 443-450.

[22] T. S. Shores, The structure of Loewy modules, J. Reine Angew. Math. 254 (1972), 204-220.

[23] W. V. Vasconcelos, Finiteness in projective ideals, J. Algebra 25 (1973), 269-278.

[24] W. Xue, Two examples of local Artinian rings, Proc. Amer. Math. Soc. 107 (1989), $63-65$.

Department of Mathematics

University of Ahvaz

Ahvaz, Iran

E-mail: karamzadeh@cua.ac.ir

Received 6 November 2003;

in revised form 1 December 2004 